\title{
Review Article \\ Revealing the Therapeutic Uses of Garlic (Allium sativum) and Its Potential for Drug Discovery
}

\author{
Azene Tesfaye (iD) \\ Department of Medical Laboratory Science, College of Medicine and Health Sciences, Arba Minch University, \\ Arba Minch, Ethiopia \\ Correspondence should be addressed to Azene Tesfaye; azet567@gmail.com
}

Received 19 October 2021; Accepted 20 December 2021; Published 30 December 2021

Academic Editor: Juei Tang Cheng

Copyright (c) 2021 Azene Tesfaye. This is an open access article distributed under the Creative Commons Attribution License, which permits unrestricted use, distribution, and reproduction in any medium, provided the original work is properly cited.

Background. Garlic is a common bulb vegetable that is used to flavor and flavor food. The plant contains biologically active components that contribute to its pharmacological properties. This paper attempts to examine the therapeutic uses and potential role in the drug development of garlic for various human diseases. Methods. To obtain crucial data and scientific knowledge about the therapeutic uses of garlic, systematic literature searches were conducted using key terms on well-known indexed platforms such as PubMed, Scopus, Web of Science, Medline, Embase, and popular search engines. Results. Garlic, which is utilized as a spice and flavoring ingredient, is found to have fundamental nutritional components. Carbohydrates, protein, fat, minerals, water, and vitamins are all found in abundance in this plant. The plant also has a high medicinal value and is used to cure a variety of human diseases. It has anti-inflammatory, rheumatological, ulcer inhibiting, anticholinergic, analgesic, antimicrobial, antistress, antidiabetes, anticancer, liver protection, anthelmintics, antioxidants, antifungal, and wound healing properties, as well as properties that help with asthma, arthritis, chronic fever, tuberculosis, runny nose, malaria, leprosy, skin discoloration, and itching, indigestion, colic, enlarged spleen, hemorrhoids, fistula, bone fracture, gout, urinary tract disease, diabetes, kidney stones, anemia, jaundice, epilepsy, cataract, and night blindness. Conclusions. The nutritional content of the plant is significant, and it has incredible therapeutic potential. The findings of this study are needed to investigate the therapeutic potential, as it may be a promising option for drug development.

\section{Introduction}

For thousands of years, humans have employed natural plant-based chemicals to cure a variety of foods [1]. Nutritional variables have an important influence on the onset of a variety of human disorders [2]. Many different diets are believed to be beneficial to human health in many cultures. Despite ethnic diversity, healthy eating habits share certain basic elements [3]. Garlic (Allium sativum L.) is a member of the Alliaceae family and, after onions, is the second most widely used Allium. It is widely produced globally and used as a spice, additive, and medicinal plant, as stated [4-7].

Garlic has a higher concentration of sulfur compounds (allicin, diallyl disulfide, S-allylcysteine, and diallyl trisulfide), which are responsible for its therapeutic properties. It is consumed either raw (fresh leaves or dried cloves) or processed (garlic oil, garlic extracts, and garlic powder), with varying chemical compositions and bioactive ingredient levels. It is long been known as a beneficial spice and a popular treatment for a variety of diseases and physiological conditions $[8,9]$.

Garlic is employed in a variety of ways as a therapeutic remedy in modern society. As a result, scientists in numerous fields are currently concentrating their efforts on determining the medicinal potential of garlic in human health $[2,10]$. The broad-spectrum therapeutic effects of garlic with low toxicity are of prime interest to researchers studying its medicinal properties [2]. Garlic extract has antibacterial, fungicidal, and viral action [11]. Several authors have complimented the chemical components of garlic for the treatment of cardiovascular disease, cancer, diabetes, blood pressure, atherosclerosis, and hyperlipidemia $[2,10$, 
<smiles>C=CCSS(=O)CC=C</smiles>

Allicin<smiles>C=CCSSSCC=C</smiles><smiles>C=CCS(=O)C[C@H](N)C(=O)O</smiles>

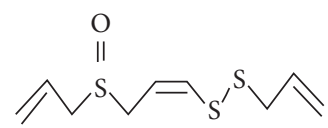

Z-Ajoene<smiles>C=CC1SC=CCS1</smiles>

2-Vinyl-4H-1,3-dithiin

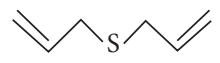

Diallyl sulfide (DAS)<smiles>C=CCSC</smiles>

Allyl methyl sulfide (AMS<smiles>C=CCS/C=C/CS(=O)CC=C</smiles>

Diallyl trisulfide

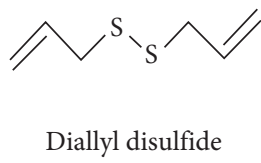

(DADS)

Figure 1: Biological active constituents and their derivatives in garlic (Allium sativum).

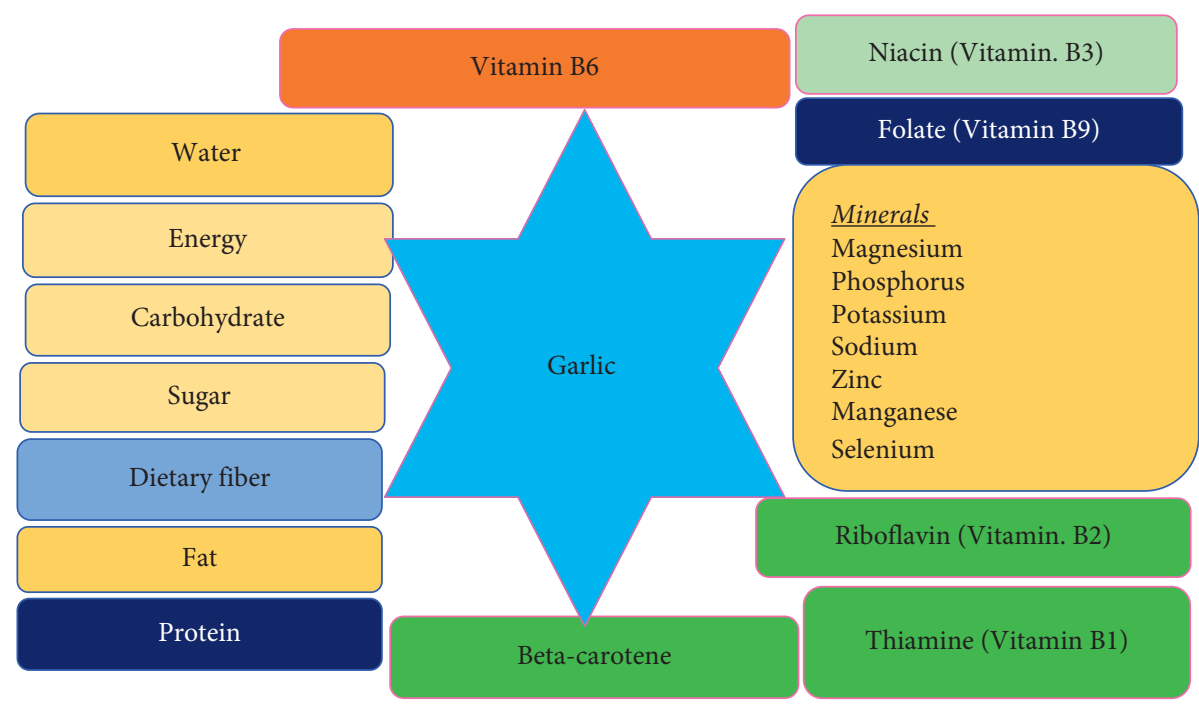

FIGURE 2: Nutritional compositions of garlic (Allium sativum).

11]. As a result, this study examines the nutritional composition and medicinal uses of garlic, as well as its potential for drug development.

\section{Methods}

2.1. Data Sources and Data Extraction. Systematic literature searches were conducted on published articles using the key terms including allicin, ajoene, Allium sativum, organosulfur compounds, and $t$ use therapies on a well-known index platform to acquire the needed information. The databases PubMed, Scopus, Web of Science, Medline, Embase, the Cochrane Library, WorldCat, Epistemonikos, and Science Direct, as well as the popular search engines (Pdf searcher.org, Google Scholar, Osun.org, and Science Direct), were used. Only 50 out of 200 published articles met the criteria for inclusion, and data extraction was carried out.

\section{Result}

3.1. Phytochemical Constituents of Garlic. Organosulfur compounds (Figure 1) such as allicin, diallyl thiosulfonate (allicin), diallyl sulfide (DAS), diallyl disulfide (DADS), diallyl trisulfide (DATS), E/Z-ajoene [12, 13], S-allylmercapto [14-16], N-acetylcysteine (NAC) [17], and S-allylcysteine (SAC) are present in garlic. Among the substances identified in garlic include peptides, steroids, terpenoids, flavonoids, and saponins [18], as well as phenols. Organosulfur compounds from raw garlic are more easily digested than those from cooked garlic [18]. There are amino acids, minerals, and enzymes such as alliinase, peroxidases, and myrosinase [9], as well as selenium, germanium, tellurium, and other trace elements in addition to sulfur compounds. Garlic polysaccharides also comprise $85 \%$ fructose, $14 \%$ glucose, and 1\% galactose [19]. 
3.2. Nutritional Values of Garlic. Garlic is used as a spice in the food industry, both fresh and dried. It keeps track of nutritionally relevant quantities (Figure 2). In addition, sugar, protein, fat, vitamins $[8,20]$, calcium, potassium, phosphorus, sulfur, iodine, fiber, and silicon are all found in garlic [21]. In addition to its flavor, it contains minerals, vitamins, and other components that are good for human health [20]. Because of its pungent smell, the plant is mostly used as a condiment and seasoning for recipes that contain both green tops and bulbs. Garlic adds flavor to dishes while also making them simpler to digest. It is highly recommended in the world's most opulent kitchens.

\subsection{Therapeutic Uses of Garlic. Because of its biologically} active component, allicin, and its derivatives, garlic has long been used as a medicine to treat a variety of illnesses and disorders including high blood pressure [8], high cholesterol, coronary artery disease [22], cancers such as colon, rectal, stomach, breast, prostate, and bladder cancers, as well as lung cancer [23], and cardiovascular diseases such as antilipemic, hypotensive, enlarged prostate (BP-hyperplasia), diabetes, osteoarthritis, hay fever (allergic rhinitis), travelers' diarrhea, preeclampsia, and cold and flu. It's also used to boost immunity and prevent and cure bacterial [24] and fungal infections [5]. The plant treats fever, cough, headache, abdominal pain, sinus congestion, gout, rheumatism, hemorrhoids, asthma, bronchitis, shortness of breath, low blood pressure, low blood sugar, high blood sugar, and snake bites [2], as well as maintaining healthy liver function, asthma, arthritis, back pain, bronchitis, chronic fever, tuberculosis, rhinitis, malaria, and stubborn skin conditions such as leprosy [25]. Garlic cloves have antilipemic (cholesterol-lowering), antihypertensive, antimicrobial, and anticancer qualities, all of which help prevent cancer cells from forming in the stomach, liver, and other human organs [2], and also help with asthma, arthritis, back pain, bronchitis, chronic fever, tuberculosis, rhinitis, malaria, stubborn skin conditions including leprosy, leucoderma, skin discoloration, and itching colic, enlarged spleen, hemorrhoids, fistula, bone fracture, gout, urinary tract disease, diabetes, and kidney stone [9]. The plant also has antibacterial and anthelmintic capabilities, as well as diuretic, digestive, and vaginal infections, platelet effects, sickle cell anemia, liver-protecting, and detoxifying properties, as well as antioxidant and radiation-protecting properties [1].

3.4. Antidiabetic Activity. In diabetic mice, garlic extract dramatically reduced serum glucose, total cholesterol, triglycerides, urea, uric acid, aspartate aminotransferase, and alanine aminotransferase while significantly increasing serum insulin [26]. It also works to lower serum glucose levels in diabetic rabbits, rats, and mice that have been induced with STZ or alloxan [27]. The volatile sulfur components in garlic are primarily responsible for its antidiabetic action. Garlic has also been demonstrated to help with insulin resistance management [28].
3.5. Antioxidant Activity. The presence of diallyl sulfide (DAS) and diallyl disulfide (DADS), as well as s-ethylcysteine (SEC) and n-acetylcysteine (NAC), protected against lipid-related oxidation by activating antioxidant enzymes. Garlic is high in antioxidants, which aid in the destruction of free radical particles that damage cell membranes and DNA, contributing to the aging process. Garlic is high in antioxidants, which assist to fight free radicals, which can damage cell membranes and DNA and speed up the aging process $[29,30]$.

3.6. Hepatoprotective Activity. Garlic is an antioxidant that can be used to treat alcoholic diseases, although it is an inefficient treatment. Marker enzymes for liver function and integrity include ALT, AST, and ALP. Lead administration resulted in a considerable rise in plasma ALT and ALP activities, as well as a reduction in plasma AST activity. The activity of ALT and ALP was greatly lowered after draining garlic, while the activity of AST was significantly elevated [30]. In rats, aged garlic and garlic diallyl sulfur compounds protected them from hepatotoxicity caused by chemicals. Aflatoxin B1 mutagenesis effects are avoided by aged-garlic extracts, which have been proven to reduce both the production and bioactivation of liver carcinogenic nitrosamines [9].

3.7. Anti-Inflammatory Activity. The anti-inflammatory properties were attributed to a reduction in the expression and production of the proinflammatory cytokines TNF- and IL-1 [31]. The presence of bioactive components such as diallyl sulfide, which is a proinflammatory cytokine that inhibits TNF and IL-1 secretion, and allyl methyl sulfide, which has been shown to stimulate anti-inflammatory cytokine and IL-10 production, could explain the regulation of proinflammatory and anti-inflammatory cytokine levels in the colon through garlic oil treatment [32].

3.8. Cardiovascular Activity. Garlic consumption lowers blood pressure, inhibits atherosclerosis, decreases serum cholesterol and triglycerides, suppresses platelet aggregation, and increases fibrinolytic activity, among other things [10]. Garlic also inhibits the formation of 3-hydroxy-3-methylglutaryl-CoA, which lowers cholesterol levels. Garlic has been found to decrease LDL oxidation, platelet aggregation, and arterial plaque development, as well as lower homocysteine, lower blood pressure, and improve microcirculation, all of which are crucial in diabetes. Garlic can also aid in preventing cognitive decline by shielding neurons from neurotoxicity and apoptosis, which helps to prevent ischemia, obsessive-compulsive disorder (OCD), and neuronal death while also boosting learning and memory retention [33].

3.9. Hyperlipidemia. Garlic inhibited liver lipogenesis and cholesterol-forming enzymes like malic acid synthetase, fatty acid synthetase, glucose-6-phosphate dehydrogenase, and 3hydroxy-3-methylglutaryl-CoA (HMG-CoA) reductase. 
Water-soluble organosulfur compounds, such as S-allyl cysteine (SAC) found in mature garlic extract and diallyl disulfide (DADS) found in garlic oil, are effective inhibitors of cholesterol formation, according to in vitro research. To avoid plaque formation, everyone should be able to cut cholesterol and reduce lipid peroxidation. Low-density lipoproteins (LDL) are suppressed and LDL resistance to oxidation is increased, according to in vitro investigations [34].

3.10. Antibacterial Activity. Garlic extract inhibits the growth of Gram-positive and Gram-negative bacteria such as Staphylococcus, Streptococcus, Micrococcus, Enterobacter, Escherichia, Klebsiella, Lactobacillus, Pseudomonas, Shigella, Salmonella, Proteus, and Helicobacter pylori. This inhibition of garlic extracts due to the presence of enzymes in allicin activity is produced by alliinase [35].

3.11. Antiviral Activity. Garlic and its sulfur components demonstrated antiviral action against coxsackievirus spp., herpes simplex virus types 1 and 2 , influenza $B$, parainfluenza virus type 3 , vaccinia virus, vesicular stomatitis virus, human immunodeficiency virus type 1 , and human rhinovirus rype 2 . In general, the sequence of a virucidal effect was as follows: ajoene $>$ allicin $>$ allyl methyl thiosulfinate $>$ methyl allyl thiosulfinate $>$ methyl allyl [23].

3.12. Antifungal Activity. Both the aqueous garlic extract and the concentrated garlic oil showed an inhibitory effect against Aspergillus [36]. Allicin showed a fungicidal effect against numerous yeasts and fungi, including Candida albicans, Cryptococcus trichophyton, Histoplasma capsulatum, and Cryptococcus neoformans [37]. Garlic has been shown to inhibit the growth of fungal diseases.

3.13. Anticancer Activity. The study looked at the effects of garlic on cell lines from leukemia, melanoma, and neuroblastoma. Garlic's flavor is characterized by allyl sulfides. In experimental carcinogenesis in various malignancies, these drugs suppress both the start and promotion stages of tumorigenesis [38]. People who consumed more garlic had a $54 \%$ decreased risk of pancreatic cancer than those who consumed fewer amounts of garlic [39]. Garlic supplements such as fresh garlic extract, matured garlic, garlic oil, and many organosulfur compounds generated from garlic have all been demonstrated to have chemopreventive benefits. The presence of organosulfur compounds in garlic is thought to be responsible for the chemopreventive effect. Free radical scavenging activity was discovered in aged garlic extract but not in fresh garlic extract. S-allyl cysteine and S-allyl mercapto-L-cysteine, the two major components in aged garlic, showed the highest free radical scavenging activity. Furthermore, several organosulfur compounds produced from garlic, such as acetylcysteine, have been shown in numerous animals to slow the growth of chemically created and transplantable cancers [40].
3.14. Antihypertensive Activity. Garlic supplements may help hypertensive people reduce their blood pressure and oxidative stress [41]. An in vitro study has proven that garlic sulfur compounds, which are produced when red blood cells convert the organic polysulfide of garlic to hydrogen sulfide, have vasoactive properties and are a recognized endogenous cardiovascular protective vascular cell-signaling molecule [3].

3.15. Antiplatelet Effect. Garlic supplements have been demonstrated to reduce cyclooxygenase activity and thromboxane $\mathrm{A} 2$ production, resulting in antiplatelet action [42]. Garlic has been shown to inhibit platelet aggregation in both in vitro and in vivo experiments. Garlic's antithrombotic impact is investigated in situ by modifying fibrinolytic activity through enhanced plasminogen activation and inhibition of thrombin production [43].

3.16. Anthelmintic Activity. In vitro, an alcoholic extract showed moderate anthelmintic efficacy against human $A s-$ caris lumbricoides. Garlic is effective in the treatment of dysentery and serves as a wormery. Garlic oil is also said to have anthelmintic properties, which means it kills all dangerous parasites in the intestines. Garlic can help you get rid of intestinal worms. Garlic's sulfurous components can help you get rid of tapeworms [31]. The allicin component of garlic has antihelmintic properties against Ascaridia galli in chickens (the main active ingredient in garlic). A. galli and Heterakis gallinarum died after being exposed to garlic oil. In both parasites, garlic extract dramatically reduced glucose uptake, glycogen content, and oxygen use [44].

3.17. Reduces Stress. In hyperglycemic rats, aged garlic extract prevents adrenal hypertrophy, hyperglycemia, and elevations in corticosterone produced by immobilization stress [8].

3.18. Sickle Cell Anemia. Because aged garlic extract (AGE) and other components of AGE, such as S-allylcysteine (SAC) and fructosyl arginine, are known to have antioxidant effects, they can prevent the production of dense cells. However, like many other garlic components, antioxidant activity has been demonstrated. The combination of aged garlic extract $(4.0 \mathrm{mg} / \mathrm{ml})$ and other helpful nutrients such as black tea extract, green tea extract, Pycnogenol, lipoic acid, vitamin E, coenzyme Q10, and carotene [45] inhibited dense cell development by 50\%. Garlic's unfavorable side effects are that garlic use can cause bad breath, body odor, nausea, vomiting, gas, weight loss, face flushing, tachycardia, disorientation, sleeplessness, and allergic responses. The entire tuber generates very little juice; yet, it is potent and can be used as an emetic in modest doses. Although garlic is generally thought to be safe, there have been cases of topical garlic burns [46]. 
3.19. Vaginal Infections. Garlic is one of the most effective antibiotics. It has bactericidal and fungicidal qualities and can kill or limit the growth of bacteria that cause vaginal discomfort, vaginitis, and vaginal flow [23]. To heal this condition, many garlic cloves were consumed. Scabies can also be treated with it. In women of childbearing age, bacterial vaginosis (BV) is one of the most common causes of vaginal discharge. Garlic can be used as a natural treatment for BV. In vitro, garlic extract reduced the development of Gram-positive and Gram-negative bacteria [23].

\section{Discussion}

Since ancient times, garlic (Allium sativum L.) has been associated with a variety of biological processes. Garlic contains many biologically active components that help it to be used in pharmaceuticals. In addition, it includes important minerals, vitamins, and proteins and is well-known as a spice or spice in continental cuisine; it also has a variety of possible pharmacological effects against a variety of lifethreatening diseases and disorders, along with this plant $[8$, 42].

Garlic is used to treat a variety of diseases around the world, including high blood pressure, infections, and snakebites, and it has also been used to ward off evil spirits and has antimicrobial effects in some cultures [47]. Garlic inhibits and destroys bacteria, fungus, and parasites, as well as lowers blood pressure, cholesterol, and sugar levels, preventing blood clotting and protecting the liver. It also has anticancer effects [8]. Garlic can also help to improve the immune system, prevent sickness, and preserve good health. It can activate the lymphatic system, which aids in the evacuation of waste from the body. Furthermore, it is a powerful antioxidant and can help protect cells from free radical damage [25]. In particular, the heart, stomach, circulation, and lungs are also aided and supported by it [23]. Garlic is an efficient natural agent in the treatment of wound infections caused by the common cold, malaria, cough, and pulmonary $\mathrm{TB}$, as well as high blood pressure, sexually transmitted diseases, mental disorders, kidney and liver problems, asthma, and diabetes [6]. Excessive alcohol use reduces oxygen radical production, resulting in a reduction in the body's normal defensive mechanisms, altered enzyme action, decreased DNA repair, decreased oxygen consumption, lipid peroxidation, and protein oxidation. Raw garlic is an antioxidant that protects tissue from oxidative stress when taken orally [48].

\section{Conclusion}

The main biologically active ingredient of garlic (allicin) and its derivatives have significant nutritional and medicinal uses around the world. It's a low-toxicity, safe, and abundant source of physiologically active chemicals. Since ancient times, all portions of the plant have been used as a spice or seasoning for sausages and salads, as well as in folk medicine. The therapeutic benefits of garlic are due to a higher concentration of sulfur compounds in the plant. As a result, more pharmacological research is needed to determine its medical efficacy in improving human health.

\section{Abbreviations \\ AGE: Aged garlic extract \\ ALP: Alkaline phosphatase \\ ALT: Alanine transaminase \\ AMS: Allyl methyl sulfide \\ AST: Aspartate transaminase \\ BV: Bacterial vaginosis \\ NAC: N-acetylcysteine \\ DAS: Diallyl sulfide \\ DADS: Diallyl disulfide \\ DNA: Deoxyribonucleic acid \\ LDL: Low-density lipoproteins \\ IL-1: Interleukin-1 \\ OCD: Obsessive-compulsive disorder \\ SEC: S-ethylcysteine \\ TNF: Tumfactor-alpha factor alpha.}

\section{Data Availability}

The data generated or analyzed during this study were included in the mother document; therefore, no additional data are available.

\section{Conflicts of Interest}

The author declares that there are no conflicts of interest concerning the publication of this article.

\section{Acknowledgments}

The author sincerely thanks everyone who has contributed, directly or indirectly, to the writing of this review. Special thanks go to the authors whose work is cited in the present manuscript. The author declares that no financial grant was used for this study and that this work was not part of the author's employment.

\section{References}

[1] J. Parekh and S. Chanda, "In vitro antimicrobial activity of Trapa natans L fruit rind extracted in different solvents," African Journal of Biotechnology, vol. 6, no. 6, 2007.

[2] L. Bayan, P. H. Koulivand, and A. Gorji, "Garlic: a review of potential therapeutic effects," Avicenna journal of phytomedicine, vol. 4, no. 1, pp. 1-14, 2014.

[3] G. A. Benavides, G. L. Squadrito, R. W. Mills et al., "Hydrogen sulfide mediates the vasoactivity of garlic," Proceedings of the National Academy of Sciences, vol. 104, no. 46, pp. 1797717982, 2007.

[4] R. A. Onyeagba, O. C. Ugbogu, C. U. Okeke, and O. Iroakasi, "Studies on the antimicrobial effects of garlic (Allium sativum Linn), ginger (Zingiber officinale Roscoe) and lime (Citrus aurantifolia Linn)," African Journal of Biotechnology, vol. 3, no. 10, pp. 552-554, 2004.

[5] K. Alare, T. Alare, and N. Luviano, "Medicinal importance of garlic and onions on autonomic nervous system," Clinical Pharmacology \& Biopharmaceutics, vol. 9, no. 204, p. 2, 2020. 
[6] J. Velíšek, R. Kubec, and J. Davídek, "Chemical composition and classification of culinary and pharmaceutical garlic-based products," Zeitschrift für Lebensmittel-Untersuchung und -Forschung A, vol. 204, no. 2, pp. 161-164, 1997.

[7] H. Amagase, "Clarifying the real bioactive constituents of garlic," Journal of Nutrition, vol. 136, no. 3, pp. 716S-725S, 2006.

[8] R. Singh and K. Singh, "Garlic: a spice with wide medicinal actions," Journal of Pharmacognosy and Phytochemistry, vol. 8, no. 1, pp. 1349-1355, 2019.

[9] A. Tesfaye and W. Mengesha, "Traditional uses, phytochemistry and pharmacological properties of garlic (Allium Sativum) and its biological active compounds," Int. J. Sci. Res. Eng. Technol, vol. 1, pp. 142-148, 2015.

[10] J. Y.-Y. Chan, A. C.-Y. Yuen, R. Y.-K. Chan, and S.-W. Chan, "A review of the cardiovascular benefits and antioxidant properties of allicin,” Phytotherapy Research, vol. 27, no. 5, pp. 637-646, 2013.

[11] B. Houshmand, F. Mahjour, and O. Dianat, "Antibacterial effect of different concentrations of garlic (Allium sativum) extract on dental plaque bacteria," Indian Journal of Dental Research: Official Publication of Indian Society for Dental Research, vol. 24, no. 1, pp. 71-5, 2013.

[12] D. Y. Yoo, W. Kim, S. M. Nam et al., "Neuroprotective effects of Z-ajoene, an organosulfur compound derived from oilmacerated garlic, in the gerbil hippocampal CA1 region after transient forebrain ischemia," Food and Chemical Toxicology, vol. 72, pp. 1-7, 2014.

[13] M. Yoo, S. Lee, S. Kim, J.-B. Hwang, J. Choe, and D. Shin, "Composition of organosulfur compounds from cool- and warm-type garlic (Allium sativum L.) in Korea," Food Science and Biotechnology, vol. 23, no. 2, pp. 337-344, 2014.

[14] S. M. B. Asdaq and M. N. Inamdar, "Pharmacodynamic and pharmacokinetic interactions of propranolol with garlic (Allium sativum) in rats," Evidence-based Complementary and Alternative Medicine, vol. 2011, 2011.

[15] Y. Liu, J. Yan, X. Han, and W. Hu, "Garlic-derived compound S-allylmercaptocysteine (SAMC) is active against anaplastic thyroid cancer cell line 8305C (HPACC)," Technology and Health Care, vol. 23, no. s1, pp. S89-S93, 2015.

[16] G. A. Souza, "N-acetylcysteine an allium plant compound improves high-sucrose diet-induced obesity and related effects," Evidence-based Complementary and Alternative Medicine, vol. 2011, 2011.

[17] G. B. Tran, S. M. Dam, and N. T. Le, "Amelioration of single clove black garlic aqueous extract on dyslipidemia and hepatitis in chronic carbon tetrachloride intoxicated Swiss Albino mice," International Journal of Hepatology, vol. 2018, Article ID 9383950, 2018.

[18] C. Torres-Palazzolo, D. Ramirez, D. Locatelli, W. Manucha, C. Castro, and A. Camargo, "Bioaccessibility and permeability of bioactive compounds in raw and cooked garlic," Journal of Food Composition and Analysis, vol. 70, pp. 49-53, 2018.

[19] H. Xue-song, "Isolation and identification of garlic polysaccharide," Food Science, p. 09, 2005.

[20] J. Velek, R. Kubec, and J. Davdek, "Chemical composition and classification of culinary and pharmaceutical garlic-based products," European Food Research and Technology, vol. 204, no. 2, pp. 161-164, 1997.

[21] C. Borek, NewportBeach, CA, Recent Advances on the Nutritional Benefits Accompanying the Use of Garlic as a Supplement, pp. 15-17, 1998.

[22] P. Mikaili, S. Maadirad, M. Moloudizargari, S. Aghajanshakeri, and S. Sarahroodi, "Therapeutic uses and pharmacological properties of garlic, shallot, and their biologically active compounds," Iranian Journal of Basic Medical Sciences, vol. 16, no. 10, pp. 15-17, 2013.

[23] M. K. Alam, M. O. Hoq, and M. S. Uddin, "Medicinal plant allium sativum-A review," Journal of Medicinal Plant Studies, vol. 4, no. 6, pp. 72-79, 2016.

[24] S. Papu, "Medicinal values of garlic (Allium sativum L) in human life: an overview," Greener Journal of Agricultural Sciences, vol. 4, no. 6, pp. 265-280, 2014.

[25] R. R. Cutler and P. Wilson, "Antibacterial activity of a new, stable, aqueous extract of allicin against methicillin-resistant Staphylococcus aureus," British Journal of Biomedical Science, vol. 61, no. 2, pp. 71-74, 2004.

[26] A. Eidi, M. Eidi, and E. Esmaeili, "Antidiabetic effect of garlic (Allium sativum L.) in normal and streptozotocin-induced diabetic rats," Phytomedicine: International Journal of Phytotherapy and Phytopharmacology, vol. 13, no. 9-10, pp. 624-629, 2006.

[27] F. T. Djankpa, "Effect of regular garlic ingestion on body weight and blood glucose: a case study in mice," International Journal of Pharmaceutical Sciences and Research, vol. 3, no. 5, Article ID 1364, 2012.

[28] R. Padiya and S. K. Banerjee, "Garlic as an anti-diabetic agent: recent progress and patent reviews," Recent Patents on Food, Nutrition \& Agriculture, vol. 5, no. 2, pp. 105-127, 2013.

[29] A. Capasso, "Antioxidant action and therapeutic efficacy of allium sativum L,” Molecules, vol. 18, no. 1, pp. 690-700, 2013.

[30] G. O. Ajayi, T. T. Adeniyi, and D. O. Babayemi, "Hepatoprotective and some haematological effects of Allium sativum and vitamin C in lead-exposed wistar rats," International Journal of Medicine and Medical Sciences, vol. 1, no. 3, pp. 064-067, 2009.

[31] G. Hodge, S. Hodge, and P. Han, "Allium sativum (garlic) suppresses leukocyte inflammatory cytokine production in vitro: potential therapeutic use in the treatment of inflammatory bowel disease," Cytometry, vol. 48, no. 4, pp. 209-215, 2002.

[32] M. Balaha, S. Kandeel, and W. Elwan, "Garlic oil inhibits dextran sodium sulfate-induced ulcerative colitis in rats," Life Sciences, vol. 146, pp. 40-51, 2016.

[33] C. Borek, "Garlic reduces dementia and heart-disease risk," Journal of Nutrition, vol. 136, no. 3, pp. 810S-812S, 2006.

[34] B. H. S. Lau, "Suppression of LDL oxidation by garlic compounds is a possible mechanism of cardiovascular health benefit," Journal of Nutrition, vol. 136, no. 3, pp. 765S-768S, 2006.

[35] S.-M. Tsao and M.-C. Yin, "In-vitro antimicrobial activity of four diallyl sulphides occurring naturally in garlic and Chinese leek oils," Journal of Medical Microbiology, vol. 50, no. 7, pp. 646-649, 2001.

[36] S. T. Pai and M. W. Platt, "Antifungal effects of Allium sativum (garlic) extract against the Aspergillus species involved in otomycosis," Letters in Applied Microbiology, vol. 20, no. 1, pp. 14-18, 1995.

[37] P. Sabitha, P. M. Adhikari, S. M. Shenoy et al., "Efficacy of garlic paste in oral candidiasis," Tropical Doctor, vol. 35, no. 2, p. 99, 2005.

[38] J. M. Chan, F. Wang, and E. A. Holly, "Vegetable and fruit intake and pancreatic cancer in a population-based casecontrol study in the San Francisco bay area," Cancer Epidemiology Biomarkers \& Prevention, vol. 14, no. 9, pp. 20932097, 2005.

[39] C. A. González, P. Guillem, A. Antonio et al., "Fruit and vegetable intake and the risk of stomach and oesophagus 
adenocarcinoma in the European Prospective Investigation into Cancer and Nutrition (EPIC-EURGAST)," International Journal of Cancer, vol. 118, no. 10, pp. 2559-2566, 2006.

[40] В. Ш. Рака, "Extract of garlic (Allium sativum) in cancer chemoprevention," Experimental Oncology, vol. 25, pp. 93-97, 2003.

[41] V. Dhawan and S. Jain, "Effect of garlic supplementation on oxidized low density lipoproteins and lipid peroxidation in patients of essential hypertension," Molecular and Cellular Biochemistry, vol. 266, no. 1, pp. 109-115, 2004.

[42] D. Chakraborty and A. Majumder, "Garlic (Lahsun)-an immunity booster against SARS-CoV-2," Biotica Research Today, vol. 2, no. 8, pp. 755-757, 2020.

[43] H. Fukao, Y. Hideki, and T. Yoh Ichi, "Antithrombotic effects of odorless garlic powder both in vitro and in vivo," Bioscience Biotechnology and Biochemistry, vol. 71, no. 1, pp. 84-90, Article ID 0612070194, 2007.

[44] A. Raza, F. Muhammad, S. Bashir, B. Aslam, M. I. Anwar, and M. U. Naseer, "In-vitro and in-vivo anthelmintic potential of different medicinal plants against Ascaridia galli infection in poultry birds," World's Poultry Science Journal, vol. 72, no. 1, pp. 115-124, 2016.

[45] S. T. Ohnishi and T. Ohnishi, "In vitro effects of aged garlic extract and other nutritional supplements on sickle erythrocytes," Journal of Nutrition, vol. 131, no. 3, pp. 1085S-1092S, 2001.

[46] T. Friedman, A. Shalom, and M. Westreich, "Self-inflicted garlic burns: our experience and literature review," International Journal of Dermatology, vol. 45, no. 10, pp. 1161-1163, 2006.

[47] T. Wolde, H. Kuma, and D. Kassahun Trueha, "Anti-bacterial activity of garlic extract against human pathogenic bacteria," Journal of Pharmacovigil, vol. 6, no. 253, pp. 2-8, 2018.

[48] V. Lobo, A. Patil, A. Phatak, and N. Chandra, "Free radicals, antioxidants and functional foods: impact on human health," Pharmacognosy Reviews, vol. 4, no. 8, pp. 118-126, 2010. 